\title{
Extranasopharyngeal Angiofibroma of the Nasal Septum in an Elderly Postmenopausal Female: A Very Rare Presentation
}

\author{
Brajpal S Tyagi
}

\begin{abstract}
Nasopharyngeal angiofibromas (NPAs) are known to occur predominantly in males around puberty and adolescence. They are mostly commonly located in the region of the nasopharynx with their origin predominantly being the sphenopalatine foramen. The term extranasopharyngeal angiofibroma (ENPA) has been mainly applied to vascular, fibrous nodules that are found to occur outside of the nasopharynx. Extranasopharyngeal angiofibromas are known to be an uncommon entity. In this report, we describe the case of a 54-year-old woman who presented to us with history of a slowly progressing nasal obstruction of the left nasal cavity due to the presence of a mass. She also gave history of sudden episodes of epistaxis on and off. Typical radiological findings were found in computed tomography (CT) that showed presence of a mass adhering to the nasal septum. Initially, biopsy was done. The tumor was then successfully removed surgically and the subsequent histopathological examination confirmed the diagnosis to be of an ENPA. In follow-up, the patient is free of symptoms.
\end{abstract}

Keywords: Extranasopharyngeal angiofibroma, Females, Nasal septum.

How to cite this article: Tyagi BS. Extranasopharyngeal Angiofibroma of the Nasal Septum in an Elderly Postmenopausal Female: A Very Rare Presentation. Int J Otorhinolaryngol Clin 2018;10(1):39-41.

\section{Source of support: Nil}

Conflict of interest: None

\section{CASE REPORT}

A 54-year-old female presented to the Otolaryngology Department of our hospital with a history of a slowly progressive left-sided nasal obstruction and bleeding on and off for the duration of past 1 year. On endoscopic examination, it showed a nasal septum deviation on the right side along with a pinkish mass adhering to the left-sided nasal septum and occupying the nasal cavity. A CT scan of the paranasal sinuses showed an inhomogeneously

\footnotetext{
Head

Department of ENT, Harsh ENT Hospital, Ghaziabad, Uttar Pradesh, India

Corresponding Author: Brajpal S Tyagi, Head, Department of ENT, Harsh ENT Hospital, Ghaziabad, Uttar Pradesh, India Phone: +919999673918, e-mail: brajpaltyagi@gmail.com
}

enhancing marked thickening involving soft tissue part of nasal septum anterosuperiorly (Fig. 1). Mild mucosal thickening involving few of the bilateral ethmoidal air cells was also noted. There was no extension of the mass found beyond the nasal cavity into the nasopharynx or into other paranasal sinuses. Intraoperatively, it was found to be firm to hard in consistency, minimal bleeding on probing, and firmly adherent to the bony nasal septum. Thereafter, the tumor was elevated with the underlying periosteum and removed under endoscopic guidance using microdebrider. A biopsy specimen of the mass was obtained (Fig. 2).
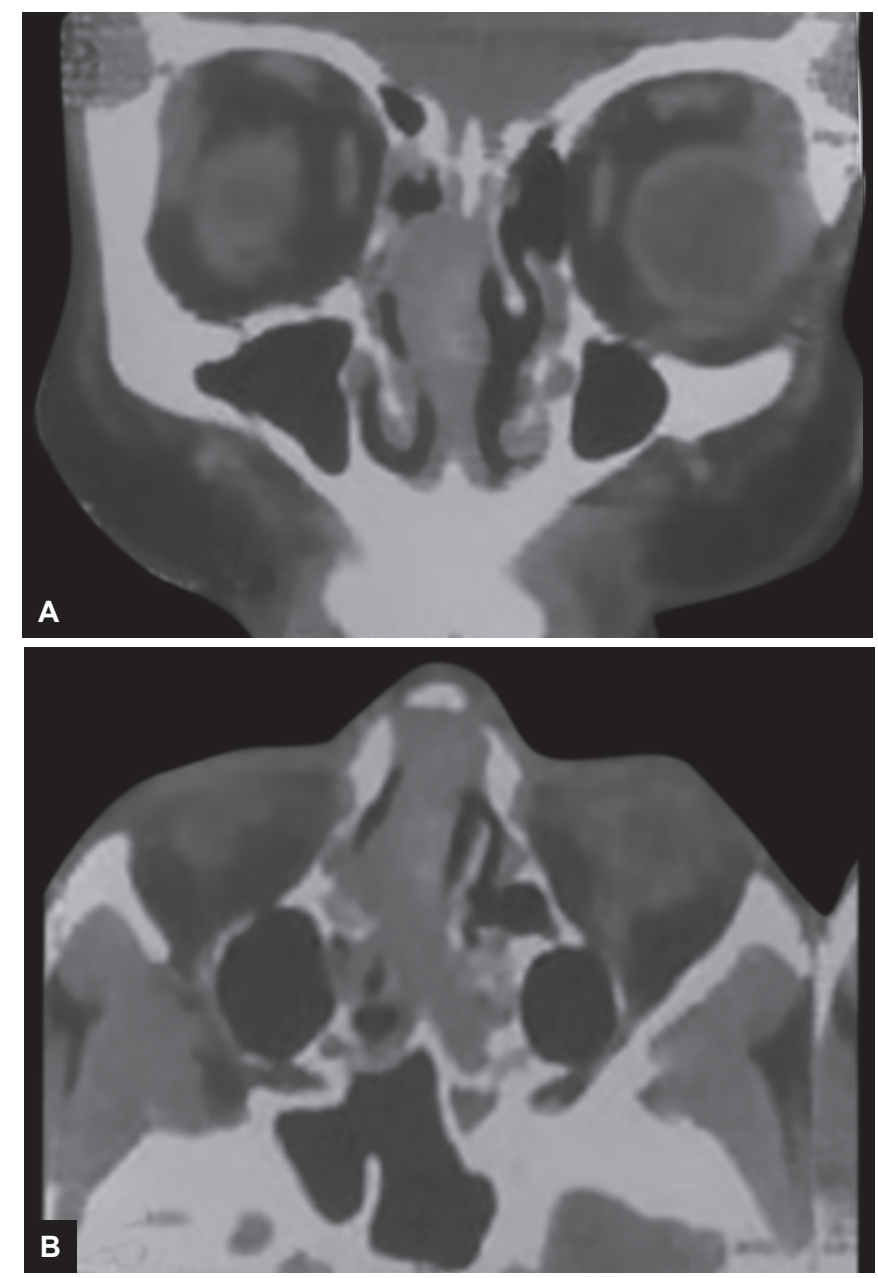

Figs $1 \mathrm{~A}$ and $\mathrm{B}$ : Computed tomography scan of the paranasal sinuses showed an inhomogeneously enhancing marked thickening, involving soft tissue part of nasal septum anterosuperiorly. (A) Coronal view; (B) axial view 


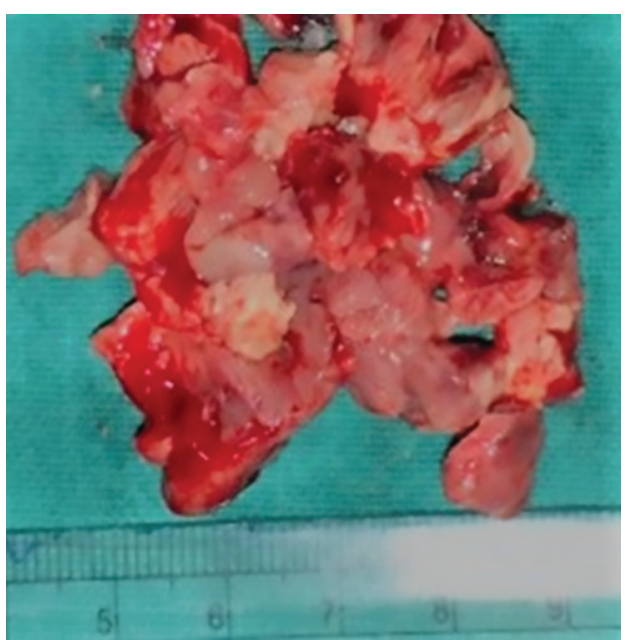

Fig. 2: Excisional biopsy specimen of a grayish pink mass

Intraoperative bleeding was found to be minimal. Antibiotic and steroid-soaked Merocel nasal pack were placed bilaterally. The packs were removed postoperatively after 48 hours and the patient was discharged without any complications. Histopathological examination revealed multiple bits of chronically inflamed, congested, and edematous nasal mucosa admixed with occasional bony spicules (Fig. 3). It also showed dense collagenization with hyalinized blood vessels and moderate chronic inflammation. No granuloma, dysplasia, or malignancy was seen in the sections examined. Features were found to be suggestive of an angiofibroma with dense collagenization.

\section{DISCUSSION}

Extranasopharyngeal angiofibroma has been mainly applied to a variety of vascular and fibrous nodules occurring anywhere away from the nasopharynx. However, ENPA does not have much in common with the NPA. As compared with NPAs, patients affected by ENPA are found to be older, females can also be involved, symptoms are prone to develop, and hypervascularity in these lesions is less common.

The case we described here fulfilled these features of ENPA. The clinical presentation depends mainly on the site of localization of the lesion and extent of the tumor margins. In those cases where it is arising from the nasal cavities, nasal obstruction and epistaxis are the major presenting symptoms. The etiology is suspected to be the presence of ectopic tissue. Battery of tests like CT scan, magnetic resonance imaging, and carotid angiogram have been found to be valuable in the diagnosis of ENPA.

The nasal septum has been found to be an extremely rare site for the origin of angiofibromas, and till date only few cases have been previously reported in the literature. ${ }^{1}$ Extranasopharyngeal sites, such as the paranasal sinuses and nasal cavity are further less frequent.



Fig. 3: Histopathology features were found to be suggestive of an angiofibroma with dense collagenization and hyalinized blood vessels and moderate chronic inflammation

Angiofibroma of the septum of the nose is extremely rare. It usually originates from the anterior septum, from the bony cartilaginous junction, or from the posterior septum. Clinical presentation is one with recurrent episodes of epistaxis and nasal obstruction secondary to a nasal mass. ${ }^{2}$

Extranasopharyngeal angiofibromas arising from the nasal septum or nasal septal angiofibromas are extremely rare; only 13 such cases have been reported in the international literature. ${ }^{3}$ A total of 65 patients with ENPAs from 16 different countries were reported in the literature. Two patients had a congenital lesion, the oldest being 78 years. A total of 48 patients were male (73\%). The maxilla was the most commonly affected site (24.6\%), with the ethmoid, nasal cavity, or septum and other sites being involved less frequently. ${ }^{4}$

In another study, angiofibroma presented in a 15-month-old boy at an atypical site anterior and medial to the lacrimal sac. ${ }^{5}$ In a rare case, a 16-year-old girl presented with a pinkish lobulated mass in her left nostril that had arisen from the anterior ethmoid sinus. The mass was removed via a lateral rhinotomy approach. ${ }^{6}$ Nomura et $\mathrm{al}^{7}$ reported a case of ENPA arising from the inferior turbinate of a 62-year-old male, which was excised using the Denker approach.

Perić et $\mathrm{al}^{8}$ suggested that immunohistochemical analysis is very important in all doubtful cases, especially in those with atypical location. Histopathological differential diagnoses for such lesions are capillary hemangioma, hemangiopericytoma, solitary fibrous tumor, and angiofibroma. To confirm the diagnosis, immunohistochemistry is done. Immunohistochemistry showing vimentin positive stromal cells, smooth muscle actin positivity in cells around vascular spaces, and CD34 positivity in vascular endothelial cells are consistent with angiofibroma.

Szymańska et a ${ }^{9,10}$ have also reported a case of ENPA presenting in the infratemporal fossa. The ENPA occurs 
more frequently in females, and accounts for 25 to $26 \%$ of all cases. They also develop in older age group than NPA. According to histopathology, ENPAs constitute a more heterogeneous group. The predominance of the vascular component in the fibrous stroma characteristic for NPA has been stated only in few patients with ENPA. Most ENPAs enhance after contrast medium injection; however, enhancement is not a constant sign. Radiological presentation of ENPA is much more variable than NPA due to their various locations. However, lack of hypervascularity on angiograms does not exclude the diagnosis of the ENPA. The treatment of choice for ENPA is surgical removal of the mass. Radiotherapy may be done for unresectable lesions.

\section{CONCLUSION}

Extranasopharyngeal angiofibroma must be kept in the differential diagnosis of nasal tumors of vascular nature and nasal septum is a rare location of these neoplasms. The clinical features of ENPA vary widely from that of NPAs. Therefore, they present as a diagnostic challenge to the clinician. A systematic evaluation and a very high index of suspicion are required in establishing the proper diagnosis and hence the treatment.

\section{REFERENCES}

1. Mohindra S, Grover G, Bal AK. Extranasopharyngeal angiofibroma of the nasal septum: a case report. Ear Nose Throat J 2009 Nov;88(11):E17-E19.
2. Hamdan AL, Moukarbel RV, Kattan M, Natout M. Angiofibroma of the nasal septum. Middle East J Anaesthesiol 2012 Feb;21(4):653-655.

3. Garcia-Rodriguez L, Rudman $\mathrm{K}$, Cogbill CH, Loehrl T, Poetker DM. Nasal septal angiofibroma, a subclass of extranasopharyngeal angiofibroma. Am J Otolaryngol 2012 Jul-Aug;33(4):473-476.

4. Windfuhr JP, RemmertS. Extranasopharyngeal angiofibroma: etiology, incidence and management. Acta Otolaryngol 2004 Oct;124(8):880-889.

5. Schick B, Kind M, Draf W, Weber R, Lackmann GM. Extranasopharyngeal angiofibroma in a 15-month-old child. Int J Pediatr Otorhinolaryngol 1998 Feb;43(1):99-104.

6. Lerra S, Nazir T, Khan N, Qadri MS, Dar NH. A case of extranasopharyngeal angiofibroma of the ethmoid sinus: a distinct clinical entity at an unusual site. Ear Nose Throat J 2012 Feb;91(2):E15-E17.

7. Nomura K, Shimomura A, Awataguchi T, Murakami K, Kobayashi T. A case of angiofibroma originating from the inferior nasal turbinate. Auris Nasus Larynx 2006 Jun;33(2):191-193.

8. Perić A, Baletić N, Cerović S, Vukomanović-Durdević B. Middle turbinate angiofibroma in an elderly woman. Vojnosanit Pregl 2009 Jul;66(7):583-586.

9. Szymańska A, Szymański M, Skomra D, SzczerboTrojanowska M. Extranasopharyngeal angiofibroma of the infratemporal fossa. Otolaryngol Head Neck Surg 2009 Mar;140(3):433-434.

10. Szymańska A,Szymański M,Morshed K,Czekajska-ChehabE, Szczerbo-Trojanowska M. Extranasopharyngeal angiofibroma: clinical and radiological presentation. Eur Arch Otorhinolaryngol 2013 Feb;270(2):655-660. 